\title{
La gamificación como estrategia para el aprendizaje de Física
}

\author{
The gamification as strategy for the learning of Physics \\ MONROY-CARREÑO, Mireya $\dagger^{*} *$ \& MONROY-CARREÑO, Patricia \\ Escuela Nacional Colegio de Ciencias y Humanidades Plantel Vallejo-UNAM
}

ID $1^{\text {er }}$ Autor: Mireya, Monroy-Carreño / ORC ID: 0000-0002-3611-8532, CVU CONACYT ID: 743139

ID $1^{\text {er }}$ Coautor: Patricia, Monroy-Carreño / ORC ID: 0000-0002-2735-7208, CVU CONACYT ID: 424764

DOI: $10.35429 / J I T C .2019 .9 .3 .1 .12$

Recibido 06 de Julio, 2019; Aceptado 25 de Septiembre, 2019

\section{Resumen}

Numerosos estudios manifiestan el desinterés o la valoración negativa que tienen los alumnos hacia el aprendizaje de la materia de física y aunque esta percepción es provocada por diversas situaciones, no se puede dejar de lado que esto incide en la motivación de los estudiantes y en consecuencia en el aprendizaje. Por esta razón el objetivo de este estudio fue el de identificar si el uso de la gamificación en las clases influye en la motivación de los jóvenes y poder así fomentar en ellos una visión positiva hacia la asignatura de física, por lo tanto en esta investigación se implementó una metodología basada en la gamificación, la cual incluye la planeación, el diseño y la aplicación de tres juegos a lo largo de un semestre en el que participaron 50 alumnos de la asignatura de Física II de la Escuela Nacional Colegio de Ciencias y Humanidades plantel Vallejo, obteniendo como resultado que la utilización de la gamificación apunta que es un elemento motivador ya que provoca una experiencia gratificante en los estudiantes.

\begin{abstract}
Numerous studies show the disinterest or the negative evaluation that students have towards the learning of the subject of physics and although this perception is provoked by diverse situations, it can't be left aside that this affects the motivation of the students and consequently in learning. For this reason the objective of this study was to identify if the use of gamification in the classes influences the motivation of young people and thus be able to encourage them a positive view of the subject of physics, therefore in this investigation implemented a methodology based on gamification which includes the planning, design and application of three games over a semester in which participated 50 students of the subject of Physics II of the Escuela Nacional Colegio de Ciencias y Humanidades plantel Vallejo, obtaining as a result that the use of gamification indicates that it is a motivating element since it provokes a gratifying experience in the students.
\end{abstract} aprendizaje de Física. Revista de Tecnologías de la Información y Comunicaciones. 2019. 3-9: 1-12

\footnotetext{
* Correspondencia del Autor (Correo electrónico: m.monroy449@ gmail.com)

$\dagger$ Investigador contribuyendo como primer autor.
} 


\section{Introducción}

La asignatura de Física es considerada por muchos estudiantes una materia difícil y aburrida, ya sea por sus definiciones abstractas o por su relación con las matemáticas. Asimismo, en ocasiones es percibida por los jóvenes como irrelevante por no tener relación con su contexto y por consiguiente estos factores podrían incidir en el bajo desempeño académico o incluso en la deserción del curso.

Sobre las bases anteriores, se deduce que se requiere una enseñanza centrada en el alumno a través del uso de metodologías activas que propicien una mayor participación y motivación de los jóvenes en la construcción de su propio aprendizaje, las cuales sean modificado y mejorando gradualmente (Rodríguez, Gómez, Bravo \& Truyol, 2019) y con ello mejorar los resultados conseguidos hasta ahora, dado que en la sociedad actual, está demostrado que los métodos tradicionales de enseñanza no han logrado el desarrollo de habilidades científicas requeridas por la población (Sanmartí \& Márquez, 2017).

Con respecto a las reflexiones anteriores la problemática es que existe una crisis educativa que perturba a todos los niveles educativos, pero quizás uno de los niveles con mayor afectación es el medio superior, cuyos indicadores de calidad educativa como la deserción y el índice de reprobación son elevados (Corzo \& Reyes, 2017).

En relación con lo anterior, en la Escuela Nacional Colegio de Ciencias y Humanidades $(\mathrm{ENCCH})$ la situación no es diferente dado que la asignatura de Física ocupa el segundo lugar en deserción y reprobación solo superado por las materias que integran el área de matemáticas.

En atención a la problemática expuesta, se implementó la gamificación como una propuesta metodológica en la ENCCH plantel Vallejo en la materia de Física, puesto que es considerada un agente motivador para el proceso de aprendizaje, ya que genera cambios positivos en el comportamiento y en la actitud de los alumnos debido a que se incorporan elementos de la dinámica del juego propiciando un ambiente agradable (Mera, 2016 y Ortiz, Jordán \& Agredal, 2018).
Dentro de este orden de ideas diversas investigaciones manifiestan los múltiples beneficios que se obtienen al introducir esta metodología en el aula, como son el incremento del interés y la creatividad, promueve el desarrollo de habilidades y actitudes positivas para el aprendizaje significativo (Oliva, 2016; López \& Domènech, 2018), dado que emplea los principios del juego que constituye una herramienta esencial para lograr aprendizajes notables y útiles para la vida (León, Martínez \& Santos, 2019, p.111).

Por lo tanto, la importancia de este estudio apunta principalmente a la construcción de tres propuestas didácticas, una para cada unidad del programa de estudios de Física II de la ENCCH basados en la gamificación, los cuales se implementaron durante un semestre con 50 alumnos de ambos turnos que cursaban el segundo año escolar.

En el mismo sentido de acuerdo con los resultados obtenidos se observaron cambios positivos hacia la percepción de los jóvenes hacia la materia de Física, pero además se observó que el uso de la gamificación en clase ofrece una serie de ventajas que conviene aprovechar no solo por el incremento de la motivación del alumno, sino también porque hace más divertida la clase y fomenta el compañerismo. Sobre las bases de las ideas expuestas los objetivos de la presente investigación son:

\section{Objetivo general}

Identificar si el uso de los recursos didácticos basados en la gamificación influye en la motivación de los jóvenes y poder así fomentar en ellos una visión positiva hacia el estudio de la asignatura de Física.

\section{Objetivos específicos}

1. Identificar el tipo de jugadores de acuerdo con la taxonomía de Bartle (target).

2. Conocer la percepción que tiene los jóvenes hacia la asignatura de Física.

3. Implementar los materiales didácticos diseñados a partir de la gamificación.

4. Evaluar los resultados obtenidos de la aplicación de los recursos didácticos. 


\section{Marco teórico}

La palabra "Gamificación" proviene del inglés gamification cuya principal característica es que incluye componentes del juego. Algunas definiciones de gamificación según ciertos autores:

1. "Es un proceso relacionado con el pensamiento del jugador y las técnicas de juego para atraer a los usuarios y resolver problemas" (Zichermann y Cunningham, 2011 p.11 citado en Quintanal, 2016, p.329).

2. "La utilización de mecanismos, la estética y el uso del pensamiento, para atraer a las personas, incitar a la acción, promover el aprendizaje y resolver problemas" (Kappa,2012, p.9 citado en Quintanal, 2016, p.329).

3. "Es aplicar estrategias (pensamientos y mecánicas) de juegos en contextos no jugables, ajenos a los juegos, con el fin de que las personas adopten ciertos comportamientos" (Ramírez, 2014, p.1 citado en Gallego, Molina, \& Llorens, 2014).

En resumen, la gamificación permite emplear elementos del juego en contextos no recreativos o lúdicos (Sierra \& Fernández, 2019) y así "hacer del juego el centro del aprendizaje" (Escaravajal \& Martín, 2019, p.98), con la finalidad de incrementar el interés y la motivación hacia el estudio de algún tema o disciplina, características esenciales del juego por lo que constituyen un recurso para apoyar el proceso de enseñanza aprendizaje (Godoy, 2019).

\section{Elementos de la gamificación}

Para que la gamificación sea exitosa se debe tener en cuenta la pirámide de los elementos de la gamificación (Ver Figura 1), puesto que su importancia reside no en la cantidad de sus componentes sino en la coherencia que exista entre ellos (García \& Hijón, 2017).

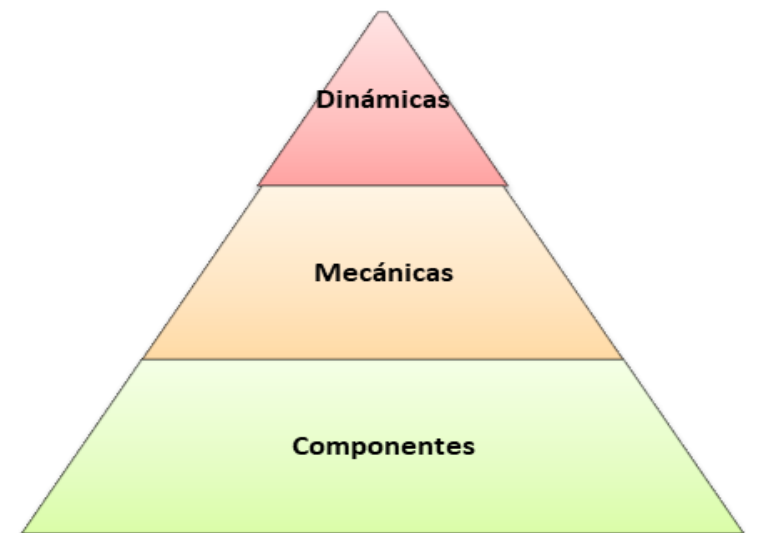

Figura 1 Pirámide de elementos de la gamificación Fuente: Werbach \& Hunter (2012)

La pirámide de los elementos de la gamificación muestra los aspectos que se deben considerar cómo son la dinámica (las restricciones, emociones, la narrativa, el progreso del jugador entre otros), la mecánica (los retos, la competición, la cooperación entre otros) y los componentes (los logros, las insignias, los niveles y puntos, entre otros).

\section{Tipos de jugadores}

Debe señalarse que otro punto que es necesario contemplar en la gamificación es el perfil de los jugadores como lo mencionan García \& Hijón (2017) es indispensable conocer las necesidades y las características de los estudiantes debido a que el diseño de las estrategias debe estar basado en el usuario y para ello sugieren el uso de la taxonomía de Bartle (pp. 47-48). Según la taxonomía de Bartle existen cuatro tipos de jugadores (Ver Figura 2).

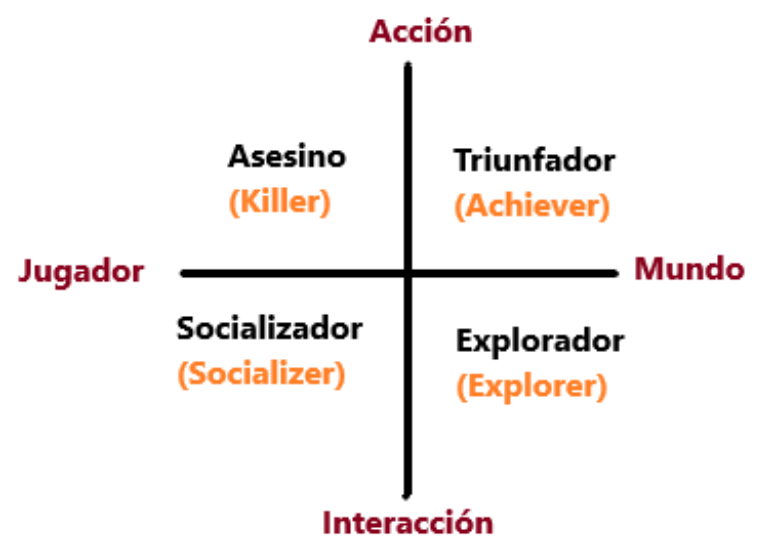

Figura 2 Gráfico de tipos de jugador Fuente: Adaptado de Bartle (2009)

Para los jugadores de tipo asesino, el objetivo es ganar, les gusta competir y les interesa los rankings y las clasificaciones; los triunfadores buscan conseguir estatus, revisan sus progresos y se comprometen por puntos, insignias, niveles. 
Los socializadores son participantes que prefieren hacer amigos y les gustan las metas en común, se interesan por los retos grupales y de cooperación y los exploradores les agrada conocer cosas nuevas y se comprometen con los niveles, retos y el desbloqueo de zonas (García \& Hijón, 2017, p. 48).

Dentro del mismo orden de ideas, el modelo propuesto de Richard Bartle presenta una prueba conformada por 36 comportamientos según las características y las motivaciones de los jugadores divididas por cuadrantes de acuerdo con el plano cartesiano, como muestra la Figura 3.

\begin{tabular}{|c|c|}
\hline 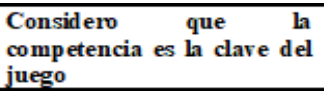 & $\begin{array}{l}\text { Considero que ha importancia } \\
\text { del juego es poder intera ctuar } \\
\text { con los otros jugadores }\end{array}$ \\
\hline $\begin{array}{l}\text { Me gustan más los juegosen } \\
\text { los que voy obteniendo } \\
\text { cosas }\end{array}$ & $\begin{array}{l}\text { Disfruto más los juegos } \\
\text { cuando hay anécdotas que } \\
\text { contar. }\end{array}$ \\
\hline $\begin{array}{l}\text { Disfruto más el juego } \\
\text { cuando gano aunque el } \\
\text { juego no sea tan divertido. }\end{array}$ & $\begin{array}{l}\text { Me gusta los juegos que sean } \\
\text { divertidos aunque no gane. }\end{array}$ \\
\hline $\begin{array}{l}\text { En juego prefiero ser el } \\
\text { primero en obtener los } \\
\text { beneficios del nivelen el que } \\
\text { me encuentro. }\end{array}$ & $\begin{array}{l}\text { En el juego prefiero explorar } \\
\text { con la finalidad de conocer los } \\
\text { detalles la historia y el } \\
\text { escenario. }\end{array}$ \\
\hline $\begin{array}{l}\text { Cuando estoy jugando me } \\
\text { involucro tanto que a veces } \\
\text { discuto con los otros } \\
\text { jugadores. }\end{array}$ & $\begin{array}{l}\text { Cedo fácilmente cuando } \\
\text { existen alguna diferencia } \\
\text { entre las opiniones de los otros } \\
\text { jugadores con tal que siga el } \\
\text { juego. }\end{array}$ \\
\hline $\begin{array}{l}\text { Si el juego no tiene un } \\
\text { ganador claro no es tan } \\
\text { bueno. }\end{array}$ & $\begin{array}{l}\text { No me interesi que haya un } \\
\text { ganador claro en el juego. }\end{array}$ \\
\hline $\begin{array}{l}\text { Me gusta recibir todo lo que } \\
\text { he recibido durante el } \\
\text { juego. }\end{array}$ & $\begin{array}{l}\text { Casi nunca reviso lo que he } \\
\text { conseguido durante el juego. }\end{array}$ \\
\hline $\begin{array}{l}\text { Me divierten los juegos que } \\
\text { me reten cada vez más }\end{array}$ & $\begin{array}{l}\text { Disfruto los juegos con } \\
\text { muchos mund os que explorar. }\end{array}$ \\
\hline $\begin{array}{l}\text { Cuando juego me agrada } \\
\text { que reconozcan mi } \\
\text { capacidad para vencer } \\
\text { retos. }\end{array}$ & $\begin{array}{llll}\begin{array}{l}\text { Cuando juego me } \\
\text { aprender y }\end{array} & \text { agrada } \\
\text { sorprendentes. } & & \text { cosas } \\
\text { sorprend }\end{array}$ \\
\hline SUMA (A) & SUMA (B) \\
\hline $\begin{array}{l}\begin{array}{l}\text { Me gustan los juegos con } \\
\text { muchos mundos por } \\
\text { explorar. }\end{array} \\
\end{array}$ & $\begin{array}{l}\text { Me gustan los juegos con } \\
\text { muchas interacciones con los } \\
\text { otros jugadores. }\end{array}$ \\
\hline $\begin{array}{l}\text { Me divierto jugando juegos } \\
\text { solitarios, por ejemplo en } \\
\text { mi teléfono celular. }\end{array}$ & $\begin{array}{l}\text { Me gustan los juegos con } \\
\text { muchas interacciones con los } \\
\text { otros jugadores. }\end{array}$ \\
\hline $\begin{array}{l}\text { Uno sabe que uno es un } \\
\text { buen jugador cuando está } \\
\text { más arriba en la tabla de } \\
\text { puntuación. }\end{array}$ & $\begin{array}{l}\text { Uno sabe que uno es un buen } \\
\text { jugador cuando los demás } \\
\text { jugadores así lo reconocen. }\end{array}$ \\
\hline $\begin{array}{l}\text { No me parecen tan } \\
\text { interesantes los juegos en } \\
\text { los que hay que discutir y } \\
\text { conversar mucho con los } \\
\text { otros jugadores }\end{array}$ & $\begin{array}{l}\text { Me gustan los juegos en los } \\
\text { que hay que discutir y } \\
\text { conversar mucho con los otros } \\
\text { jugadores. }\end{array}$ \\
\hline $\begin{array}{l}\text { Disfruto mucho cuando en } \\
\text { un juego me premian con } \\
\text { cosisespeciales }\end{array}$ & $\begin{array}{l}\text { Disfruto mucho cuando en un } \\
\text { juego me premian } \\
\text { concediénd ome un estatus } \\
\text { superioralde los demás. }\end{array}$ \\
\hline $\begin{array}{l}\text { No me gusta que me ayuden } \\
\text { cuando no puedo superar } \\
\text { un reto en un juego. }\end{array}$ & $\begin{array}{l}\text { Me gusta que me ayuden } \\
\text { cuando no puedo superar un } \\
\text { reto en un juego. }\end{array}$ \\
\hline $\begin{array}{l}\text { Cuando termino un juego } \\
\text { quiero jugar } \\
\text { inmedia tamente el siguiente } \\
\text { nivel o pasar a otro juego } \\
\text { que sea más retador. }\end{array}$ & $\begin{array}{l}\text { Cuando termino un juego me } \\
\text { gusta pasar un tiempo } \\
\text { comentando lo que sucedio. }\end{array}$ \\
\hline $\begin{array}{l}\text { Suelo ser quien se rige más } \\
\text { estrictamente a las reglas de } \\
\text { un juego. }\end{array}$ & $\begin{array}{l}\text { Suelo ser permisivo cuando se } \\
\text { trata de dejar que las reghas } \\
\text { del juego se interpreten de } \\
\text { otras maneras. }\end{array}$ \\
\hline $\begin{array}{l}\text { El juego es para pensar en } \\
\text { otra cosa y poner a prueba } \\
\text { lashabilidades }\end{array}$ & $\begin{array}{l}\text { El juego es una excusa para } \\
\text { pasar tiempo con los amigos. }\end{array}$ \\
\hline SUMAC & SUMA D \\
\hline
\end{tabular}

Figura 3 Test de personalidad del jugador Fuente: Bartle (2005)

ISSN 2531-2200

ECORFAN® Todos los derechos reservados

\section{Método y materiales}

Este estudio se realizó para la Escuela Nacional Colegio de Ciencias y Humanidades (ENCCH), Plantel Vallejo cuya población es de 3,800 alumnos que estudiaban la materia de Física II; para ello se desarrolló una técnica de muestreo no probabilístico de tipo consecutivo en 50 alumnos de cuarto semestre entre las edades de 15 a 17 años cuya muestra fueron 28 (56\%) hombres y 22 (44\%) mujeres. Asimismo, el $48 \%$ de los estudiantes pertenecían al turno vespertino.

En términos metodológicos esta investigación se ubicó en un enfoque cualitativo en el que se realizó un análisis descriptivo, a través de la recolección de datos por medio de test, encuestas y mediante observación directa, la cual se utilizó para indagar las percepciones de los alumnos hacia la asignatura de física, por lo tanto no se consideró el planteamiento de una hipótesis, dado que la finalidad de un estudio cualitativo es "reconstruir" la realidad, tal como la observan los actores de un sistema social en otras palabras no se pretende reducir la investigación en partes o en variables, debido a que la motivación y la percepción de los alumnos hacia la materia de física es de carácter multifactorial, por lo que se requiere estudiar la situación a partir de una visión holística término que precisa considerar el "todo" (Hernández, Fernández \& Baptista, 2014, p.9).

En el mismo sentido, la investigación se realizó en las siguientes etapas: Diagnóstico durante esta fase se implementó la prueba de Bartle y una encuesta el primer instrumento para identificar los tipos de jugadores que existían en el grupo y el segundo para conocer la percepción de los jóvenes hacia la materia de física.

La planeación, estuvo en base al programa estudios de Física II y a los perfiles del jugador de los alumnos e intereses; por lo tanto se diseñaron tres recursos didácticos basados en la gamificación uno para cada unidad del programa de estudios para ello se consideraron los aprendizajes que desean abordar en clase y así definir las misiones, los avatares, los premios, los logros y los niveles para que estos sean congruentes con lo que se desea alcanzar con los estudiantes y la implementación que se aplicó en 50 alumnos que cursaban la asignatura de Física II, pertenecientes a dos grupos uno del turno matutino y el otro del vespertino. 


\section{Diagnóstico}

La identificación de los tipos de jugadores (asesinos, triunfadores, socializadores y exploradores), se empleó el test de Bartle el cual consta de cuatro cuadrantes y 36 comportamientos (Ver la Figura 3), con el propósito de conocer los intereses y características de los estudiantes y con esta información proponer el ranking, las misiones, las insignias, la dinámica del juego, entre otros aspectos, esta prueba se aplicó durante la primera clase del curso de Física II.

Asimismo, se aplicó un instrumento de evaluación (encuesta) "percepción de los estudiantes hacia la asignatura de física" que consta de 13 ítems para identificar las actitudes y motivaciones de los jóvenes, diseñada a partir de instrumentos elaborados por trabajos de Cuevas, Hernández, Sampieri \& Mendoza (2016) y Barca, Porto, Santaroom \& Barca (2005). Además, se diseñó empleando la escala de Likert con la siguiente valoración 1) Muy en desacuerdo, 2) En desacuerdo, 3) Indeciso, 4) De acuerdo y 5) Muy de acuerdo; a partir de los criterios expuestos en los trabajos de García, Aguilera \& Castillo (2011) y Vallejo (2011).

Para la realización de la encuesta se utilizó un formulario de Google Forms. Cabe destacar que fue aplicada de forma anónima con el fin de que el estudiante se sintiera cómodo y con ello respondieran de forma veraz.

\section{Planeación}

En esta etapa se consideró la información obtenida de la fase del diagnóstico y de las unidades del programa de estudio de Física II de la ENCCH (ENCCH-UNAM, 2016):

\section{Electromagnetismo principios $\mathrm{y}$ aplicaciones. \\ 2. Ondas: mecánicas y electromagnéticas. \\ 3. Introducción a la física moderna y contemporánea.}

De igual modo, se examinó los aprendizajes que se debían de lograr con los alumnos en cada unidad para que cada elemento que conformará el recurso didáctico fuera congruente, para ello se implementaron pequeños experimentos exploratorios en el grupo con el propósito de establecer ciertas variaciones y requerimientos que se podrían presentar en los grupos.
Sobre las bases anteriores, antes de imprimir los juegos, las tarjetas o cualquier otro elemento que conformara el material didáctico, primero se realizaron en hojas de colores, papel bond, cartulina y en cajas de cartón, para posteriormente hacer los cambios pertinentes y pasar al diseño formal para que estos recursos fueran llamativos para los alumnos.

\section{Diseño}

En la fase de diseño se construyeron tres recursos didácticos empleando las características de gamificación, es decir, se estableció primero la misión u objetivo, las reglas de participación y la narrativa de los materiales para introducir a los jóvenes a la dinámica, posteriormente se definieron las recompensas, los niveles, los avatares y los estatus y por último establecer algunos los desafíos que pueden variar según la identidad del grupo. A continuación, se detalla cada recurso didáctico diseñado:

\section{Primer recurso: Circuito eléctrico}

En este caso el recurso didáctico es un juego de mesa que simula un circuito eléctrico con una batería y tres resistencias (Ver Figura 4) que atiende a la primera unidad del programa de estudios de Física II.

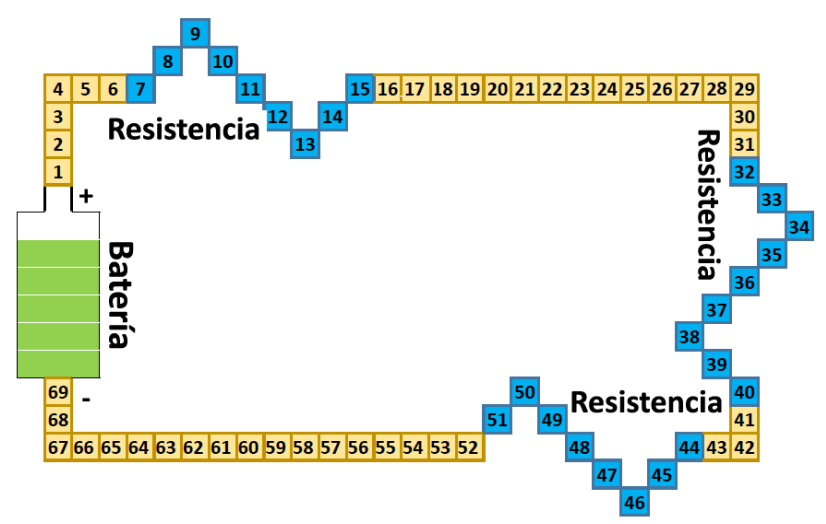

Figura 4 Tablero que simula un circuito eléctrico Fuente: Elaboración Propia

Los aprendizajes que se desean alcanzar son:

- Comprender las diferencias entre las partículas subatómicas (electrón, protón y neutrón) que conforman el átomo.

- Identificar los componentes básicos y la funcionalidad de un circuito eléctrico, asimismo los fenómenos electromagnéticos. 
En base con lo anterior y considerando la pirámide de los elementos de la gamificación y los resultados obtenidos de la etapa del diagnóstico, la dinámica de este recurso es:

Los equipos inician del polo positivo de la batería con el propósito de recorrer el circuito, la mecánica del juego se introduce en dos niveles con las siguientes misiones:

Misión 1. Convertirse en protónflash, en otras palabras, en una carga positiva, para ello los equipos deben de acabar una vuelta en el circuito.

Misión 2. Convertirse en un electroflash, es decir en una carga eléctrica negativa, en este caso el equipo tendrá que haber recorrido dos vueltas al circuito.

Dentro del mismo orden de ideas en la Figura 5 se muestra las fichas que representan un electroflash, protónflash y neutroflash. Cabe agregar que electroflash se simboliza de menor tamaño dado que es la partícula subatómica más liviana que conforma el átomo.

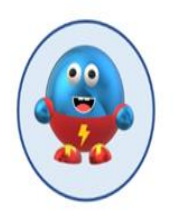

Electroflash
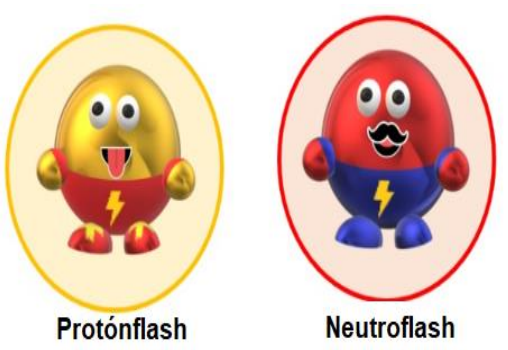

Física 5 Representación de las fichas de electroflash, protónflash y neutroflash

Fuente: Elaboración Propia

Los equipos podrán avanzar a partir de la elección de tres tipos de cartas clasificadas por el número de casillas que permiten avanzar sus valores son de 2, 4, 6 casillas respectivamente, el valor de la carta está en función de la dificultad de la pregunta.

A continuación, se explica brevemente las reglas de este recurso:

1. Un integrante de cada equipo lanzará un dado para determinar el orden de participación. El equipo que obtenga el puntaje más alto será el primero en escoger una tarjeta y así sucesivamente, en caso de empate tendrán que tirar de nuevo el dado.
2. Por equipos decidirán el valor de la tarjeta que tendrán que contestar, en otras palabras, ellos determinan que tan rápido desean recorrer el circuito eléctrico.

3. Si el equipo contesta de manera correcta podrá avanzar el número de casillas que marca la tarjeta, si la respuesta es equivocada se mantiene en el mismo lugar. Si algún otro equipo conoce la respuesta correcta podrá robar el turno de sus compañeros.

4. Iniciarán siendo un neutroflash en este caso avanzarán según el puntaje que marca la tarjeta.

5. Cuando un equipo realice la vuelta completa automáticamente se convertirá en protónflash y con ello avanzará el doble de casillas que marca la tarjeta ósea 4,8 y 12 casillas.

6. Al terminar la segunda vuelta su estatus cambiará a electroflash y por consiguiente avanzará el triple de lo que indique la tarjeta es decir 6,12 y 18 casillas.

7. Cuando un equipo se encuentre en la zona de las resistencias solo podrá avanzar la mitad del valor que muestra la tarjeta.

8. Ganará el primer equipo que se convierta en electroflash (carga eléctrica negativa) y concluya una vuelta.

\section{Segundo recurso didáctico: Cuerdas y resortes}

El segundo recurso didáctico es para la unidad de "Ondas: mecánicas y electromagnéticas", el cual está inspirado en el principio del juego de serpientes y escaleras, las cuales fueron sustituidas por cuerdas y resortes como se muestra en la Figura 6.

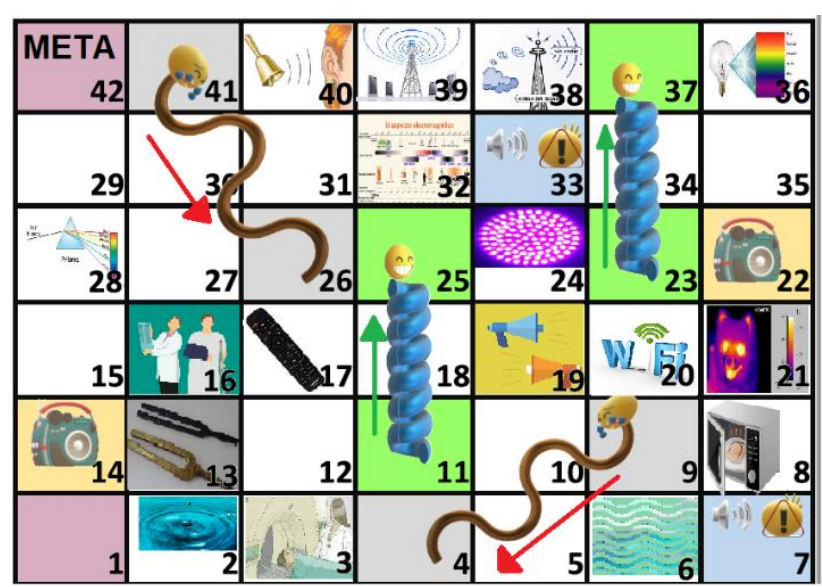

Figura 6 Tablero de cuerdas y resortes para ondas Fuente: Elaboración Propia 
Los aprendizajes que se pretenden abordar con este recurso son:

- "Identificar las magnitudes que caracterizan al movimiento ondulatorio. Identificar a las ondas como una forma en que se propaga la energía en un medio material o en el vacío.

Diferenciar las ondas mecánicas de las ondas electromagnéticas.

Describir cualitativamente cómo se generan las ondas electromagnéticas.

Comprender algunas de las aplicaciones de los fenómenos ondulatorios relacionados con la ciencia, la tecnología y la sociedad" (ENCCH-UNAM, 2016, pp.37-38).

En esta propuesta didáctica se representa la estética fundamentalmente en la narrativa o la historia que se contará al inicio de la actividad para interesar al joven en la dinámica (Ver Figura 7).

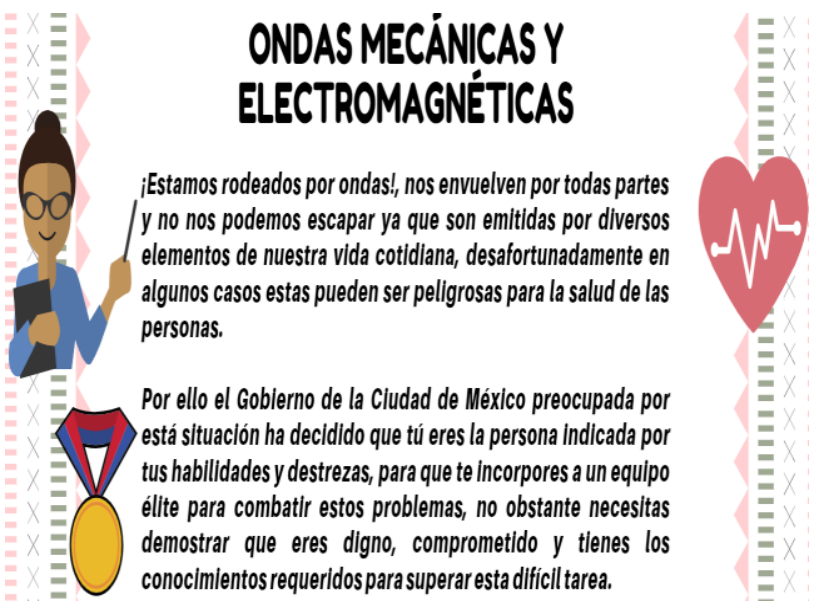

Figura 7 Narrativa para introducir la actividad de cuerdas y resortes

Fuente: Elaboración Propia

En el caso particular de este recurso la dinámica del juego es por equipo y consistirá en tirar un dado primero para designar el turno que le corresponde y después para avanzar en el tablero.

Para que un equipo avance tendrá que lanzar el dado y el número que obtenga son los lugares que avanzará siempre y cuando responda de manera correcta si la imagen de la casilla a la que va a llegar es una onda de tipo mecánica o electromagnética, en caso contrario se quedará en el mismo sitio en el que se encontraba.

Cabe agregar que el tablero tiene otros elementos que se explicarán en la Tabla 1.

\begin{tabular}{|c|l|}
\hline Elemento & \multicolumn{1}{|c|}{ Significado } \\
\hline & $\begin{array}{l}\text { Cuando algún equipo caiga en una } \\
\text { casilla donde este una carita } \\
\text { llorando unida a una cuerda; el } \\
\text { jugador tendrá que descender a la } \\
\text { casilla en la que se encuentra el final } \\
\text { de esta. }\end{array}$ \\
\hline $\begin{array}{l}\text { Cuando algún equipo caiga en una } \\
\text { casilla donde este el inicio de un } \\
\text { resorte avanzará hasta donde se } \\
\text { encuentre una carita feliz unida al } \\
\text { mismo resorte. }\end{array}$ \\
\hline $\begin{array}{l}\text { Cuando algún equipo caiga en una } \\
\text { casilla donde este la imagen de un } \\
\text { radio, este tendrá la oportunidad de } \\
\text { tirar nuevamente el dado. }\end{array}$ \\
\hline imagen & $\begin{array}{l}\text { El equipo que caiga en una casilla de } \\
\text { ruido excesivo perderá un turno. }\end{array}$ \\
\hline blanca. & $\begin{array}{l}\text { Si algún equipo cae en una casilla } \\
\text { sin imagen, el profesor le realizará } \\
\text { una pregunta a uno de los } \\
\text { integrantes del equipo, si la contesta } \\
\text { correctamente ganará una insignia, } \\
\text { la cual podrá cambiar al finalizar el } \\
\text { juego por una participación que es } \\
\text { válida para todos los integrantes del } \\
\text { equipo. }\end{array}$ \\
\hline
\end{tabular}

Tabla 1 Significado de los componentes del recurso didáctico del tablero de "Cuerdas y resortes"

Fuente: Elaboración Propia

El juego termina cuando uno de los equipos alcance la meta (casilla 42) y de acuerdo con el orden de casillas en las que se encuentre el resto de los equipos es la posición en la que se asignarán los lugares.

Cabe mencionar que debe de caer exactamente en la casilla 42 en caso contrario de que el número que salga en el dado sea mayor al que necesitaba para llegar a esta casilla entonces debe de retroceder los lugares que le sobraron.

\section{Tercer recurso didáctico: Fisipoly}

Fisipoly es un juego basado en el monopolio y está enfocada en la unidad 3 "Introducción a la física moderna y contemporánea", en este caso los inmuebles que los alumnos podrán comprar o vender están inspirados en las universidades o centros de investigación en las que estudiaron o trabajaron algunos premios Nobel en Física contemporánea (Ver Figura 8). 


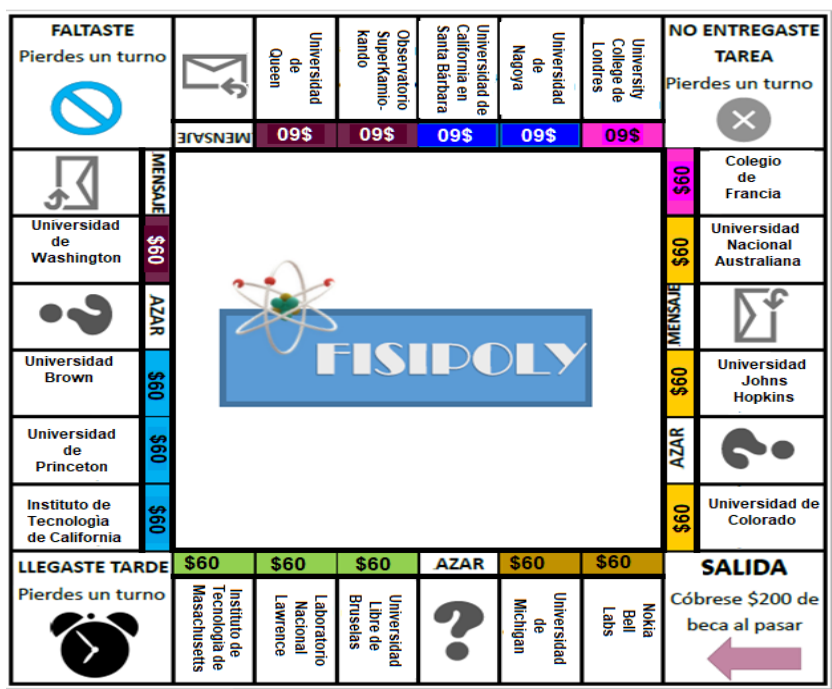

Figura 8 Tablero de Fisipoly Fuente: Elaboración Propia

El aprendizaje que se pretende lograr con esta propuesta es:

"Reconocer la importancia de las contribuciones de la física contemporánea al desarrollo científico y tecnológico" (ENCCHUNAM, 2016, p.42). Asimismo, este juego considera la temática de cuantización de la materia y energía.

El tablero consta de 28 casillas, de las cuales 18 corresponden a las instituciones o universidades en las que estudiaron o que tienen relación con los ganadores de los premios Nobel, la dinámica sigue las reglas del monopolio con algunos pequeños cambios como son la narrativa o la historia de inicio de la actividad como se muestra en la Figura 9.

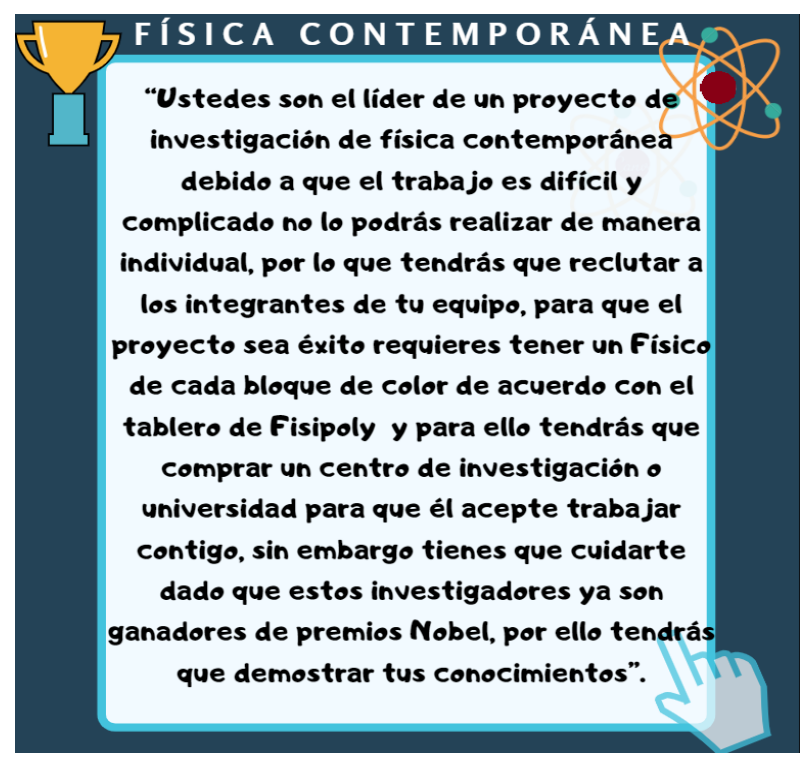

Figura 9 Narrativa de la propuesta didáctica Fisipoly Fuente: Elaboración Propia
La misión de la propuesta didáctica es que los alumnos deben convertirse en dueños de por lo menos de un instituto o universidad de cada color, cuando ellos adquieran dicha posesión el banco le entregará un título de propiedad en la que contiene el nombre del Físico que estudio o trabajo en dicha institución y cuál fue su aportación para ganar el premio Nobel.

Para que los alumnos puedan comprar una propiedad deben de haber caído en la casilla y contestado de forma correcta una pregunta referente a física contemporánea y cuyo inmueble no tenga dueño.

Los jugadores mantendrán sus posesiones mientras contesten de manera correcta las preguntas que le hagan, en caso contrario otro participante tendrá la oportunidad de responder y si es correcta, entonces se hará un contrato de compraventa para transferir la propiedad al nuevo dueño.

Cabe agregar que en este juego existen estatus según el número de propiedades que tengan, cuya clasificación es:

1 propiedad $=$ Candidato $\mathrm{a}$ investigador

2 propiedades $=$ Investigador nivel I

3 propiedades $=$ Investigador nivel II

4 propiedades $=$ Investigador III

5 propiedades $=$ Investigador emérito

En referencia a lo anterior, dependiendo de la categoría de investigador que alcance el estudiante será la recompensa que obtendrá.

La razón de las actividades anteriores es debido a que los estatus, las recompensas, son factores esenciales para una gamificación exitosa.

\section{Implementación}

Las propuestas didácticas se utilizaron a lo largo de todo el semestre 2019-2 (enero-mayo), con un total de 50 alumnos, 26 estudiantes pertenecían al turno matutino y 24 jóvenes al turno vespertino.

Cabe agregar, que la etapa del diagnóstico y el diseño de los recursos didácticos se realizaron durante el semestre del 2019-1 (agosto-noviembre del 2018). 
Debido a que cada recurso estuvo centrado para cada unidad de aprendizaje del programa de estudio de Física II, generalmente se empleaba como una actividad de cierre, con la finalidad de evaluar los aprendizajes alcanzados por los estudiantes.

\section{Resultados y discusión}

En la etapa de diagnóstico se emplearon dos instrumentos de evaluación:

1. Una prueba para conocer el perfil del jugador (Figura 3).

2. Una encuesta para conocer la percepción de los alumnos acerca de la materia de física y sus motivaciones.

\section{Prueba de Bartle}

Después de la aplicación de la prueba de Bartle se procedió a sistematizar la información de las respuestas de cada alumno ubicándolas en los ejes del plano cartesiano para así conocer el cuadrante en el que se localizaba el mayor número de respuestas y con ello identificar su comportamiento en el juego (Ver Figura 2), obteniendo así la Tabla 2 que muestra los resultados por género y la Gráfica 1 por porcentaje.

\begin{tabular}{|l|r|r|r|}
\hline \multicolumn{1}{|c|}{ Clasificaciones } & Femenino & Masculino & Total \\
\hline Asesinos & 2 & 5 & 7 \\
\hline Triunfadores & 9 & 10 & 19 \\
\hline Socializadores & 8 & 7 & 15 \\
\hline Exploradores & 3 & 6 & 9 \\
\hline Número total de estudiantes & 22 & 28 & 50 \\
\hline
\end{tabular}

Tabla 2 Resultados obtenidos del perfil de jugadores por género

Fuente: Elaboración Propia

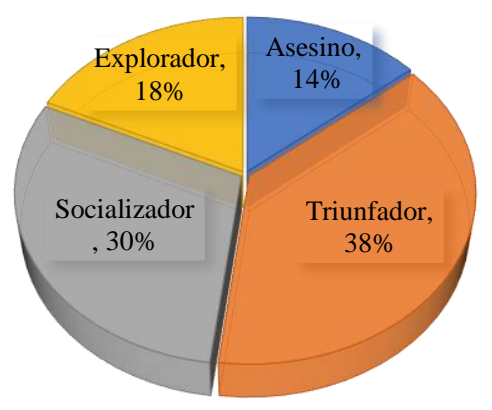

Gráfica 1 Porcentaje de alumnos de acuerdo con su perfil de jugador

Fuente: Elaboración Propia

Los resultados que muestra la Gráfica 1 se percibe que los comportamientos que predominan en el grupo de estudio son los triunfadores y los socializadores.
Por ello los recursos didácticos se diseñaron en base a niveles, estatus y trabajó en equipo para propiciar una red social.

\section{Encuesta. "Percepción de los estudiantes hacia la asignatura de física"}

El segundo instrumento de evaluación se aplicó al inicio y al final del semestre, el cual está conformado por un total de 13 items, a partir de la escala de Likert (Valor máximo 5 y valor mínimo 1), para una muestra de 50 estudiantes, obteniendo así la Tabla 3 y la Tabla 4.

\begin{tabular}{|c|c|}
\hline ÍTEMS & $\overline{\boldsymbol{x}}$ \\
\hline 1. La materia de física es aburrida. & 4.20 \\
\hline $\begin{array}{l}\text { 2. La asignatura de física es importante para conocer } \\
\text { el mundo que nos rodea. }\end{array}$ & 4.35 \\
\hline 3. La física nos ayuda a resolver problemas. & 3.80 \\
\hline 4. Las clases de física son divertidas. & 2.15 \\
\hline 5. Aprender física es interesante. & 1.57 \\
\hline 6. Es importante aprender la materia de física. & 4.17 \\
\hline 7. Me gustan los temas que se abordan en la clase de física. & 3.08 \\
\hline $\begin{array}{l}\text { 8. Lo importante para mí es conseguir buenas } \\
\text { calificaciones. }\end{array}$ & 4.31 \\
\hline $\begin{array}{l}\text { 9. Prefiero estudiar los temas que se me hacen } \\
\text { interesantes, aunque sean difíciles. }\end{array}$ & 4.53 \\
\hline $\begin{array}{l}\text { 10. Reconozco que estudio para acreditar y no para } \\
\text { aprender. }\end{array}$ & 3.80 \\
\hline 11. Se me facilita el estudio de la física. & 1.67 \\
\hline $\begin{array}{l}\text { 12. Considero que aprender física es importante para } \\
\text { mi carrera. }\end{array}$ & 3.75 \\
\hline $\begin{array}{l}\text { 13. Mis malas calificaciones son un reflejo de que la } \\
\text { materia es difícil. }\end{array}$ & 2.41 \\
\hline
\end{tabular}

$\bar{x}=$ Frecuencia media

Tabla 3 Frecuencia media de las percepciones y motivaciones de los estudiantes con respecto a la materia de física al inicio del semestre 2019-2

Fuente: Elaboración Propia

\begin{tabular}{|c|c|}
\hline ÍTEMS & $\bar{x}$ \\
\hline 1. La materia de física es aburrida. & 2.80 \\
\hline $\begin{array}{l}\text { 2. La asignatura de física es importante para conocer } \\
\text { el mundo que nos rodea. }\end{array}$ & 4.45 \\
\hline 3. La física nos ayuda a resolver problemas. & 3.70 \\
\hline 4. Las clases de física son divertidas. & 3.90 \\
\hline 5. Aprender física es interesante & 4.01 \\
\hline 6. Es importante aprender la materia de física. & 4.38 \\
\hline 7. Me gustan los temas que se abordan en la clase de física. & 3.08 \\
\hline $\begin{array}{l}\text { 8. Lo importante para mí es conseguir buenas } \\
\text { calificaciones. }\end{array}$ & 4.26 \\
\hline $\begin{array}{l}\text { 9. Prefiero estudiar los temas que se me hacen } \\
\text { interesantes, aunque sean difíciles. }\end{array}$ & 4.31 \\
\hline $\begin{array}{l}\text { 10. Reconozco que estudio para acreditar y no para } \\
\text { aprender. }\end{array}$ & 3.69 \\
\hline 11. Se me facilita el estudio de la física. & 3.92 \\
\hline $\begin{array}{l}\text { 12. Considero que aprender física es importante para } \\
\text { mi carrera. }\end{array}$ & 3.50 \\
\hline $\begin{array}{l}\text { 13. Mis malas calificaciones son un reflejo de que la } \\
\text { materia es difícil. }\end{array}$ & 1.57 \\
\hline
\end{tabular}

Tabla 4 Frecuencia media de las percepciones y motivaciones de los estudiantes con respecto a la materia de física al final del semestre 2019-2

Fuente: Elaboración Propia 
Los resultados anteriores puede ser efecto de que a lo largo de los años van desarrollando actitudes y emociones con respecto a la enseñanza de las ciencias dependiendo de sus desempeños académicos y otros estímulos (Murillo, Carrasquero \& Cañada, 2018).

Considerando las puntuaciones de la frecuencia media expresadas en la Tabla 3 y 4 , se puede definir que los valores superiores a 3 expresan que están de acuerdo (entre mayor sea este valor y se acerque a 5 implica que el grupo está totalmente de acuerdo); si el valor es menor a 3 entonces se estima que existe un grado de desacuerdo por parte del grupo estudiado y si el valor es 3 o cercano significa que el grupo está indeciso o en un punto intermedio.

Desde la perspectiva anterior se realizó un análisis comparativo entre las respuestas de los estudiantes al inició y al finalizar el semestre del 2019-2, obteniendo así la Gráfica 2.

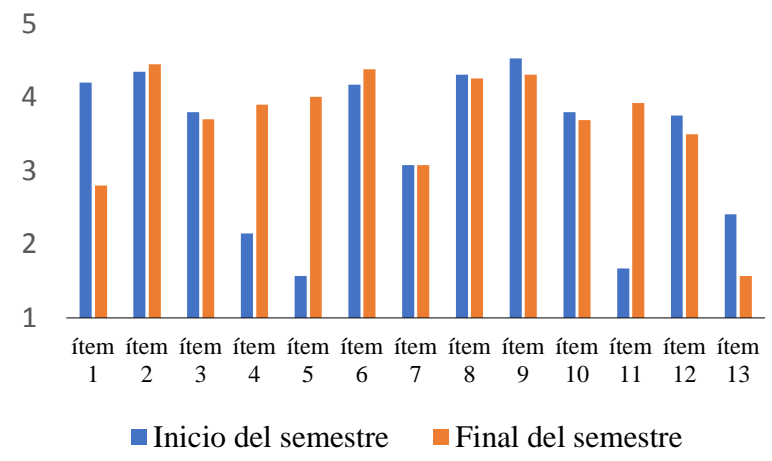

Gráfica 2 Comparación de la frecuencia media de la percepción de los estudiantes hacia la materia de física al inicio y al final del semestre

Fuente: Elaboración Propia

Conviene destacar de la Gráfica 2 la diferencia entre las respuestas iniciales y finales después de la aplicación de las propuestas didácticas principalmente en las preguntas 1, 4, 5,11 y 13, en las cuales la percepción hacia la materia de Física se modificó en un mayor grado.

La modificación de las respuestas en los alumnos puede deberse a que la gamificación emplea elementos de los juegos los cuales tienen la cualidad de estimular tres aspectos la espontaneidad, la motivación y la imaginación, ya que fomenta la participación libre de los alumnos haciendo uso de sus destrezas y habilidades que los motivan en sus aprendizajes (Calderón, 2013 citado en Montero, 2017).
Por lo cual estos factores podrían influir en las respuestas de los jóvenes como indica Gallardo \& Gallardo (2018) los juegos incrementan la atención, la memoria, estimula la alegría, el autoconcepto, la autoestima y el crecimiento personal (p.49).

En la misma orientación se resalta que diversas investigaciones han mencionado que uno de los principales factores que existen en el fracaso escolar es la apatía y la desmotivación por parte del alumnado, por lo tanto, el contexto educativo se encuentra en una constante búsqueda de alternativas que permitan mejorar esta situación y la gamificación parece ser una propuesta innovadora para usar en clase, pero como en todos los casos se debe utilizar de manera informada y supervisada por el docente.

\section{Conclusiones}

El objetivo de esta investigación fue el de identificar si el uso de los recursos didácticos basados en la gamificación influye en la motivación de los jóvenes y poder así fomentar en ellos una visión positiva hacia el estudio de la asignatura de física y al juzgar por los resultados obtenidos se puede inferir que se observaron cambios positivos en la percepción de los estudiantes para esta materia, entre los que se destacan que al final del semestre un mayor número de alumnos considera que la asignatura es divertida e interesante y que les resulta fácil estudiarla.

Cabe agregar que en los grupos que se implementó estos recursos se halló un mayor porcentaje de alumnos que lograban aprendizajes significativos y lo relacionaban con el estatus logrado e incluso cuestionaban cuando se iba a implementar de nuevo la actividad ya que estaban estudiando para alcanzar un nuevo nivel. Por lo que se concluye que los recursos diseñados mediante la gamificación pueden influir en la motivación de los alumnos y en consecuencia en su percepción hacia el estudio de Física, cuya materia ha estado estigmatizada por ser difícil y aburrida.

Por último, es conveniente anotar que la implementación de la gamificación en el aula ofrece una serie de ventajas que conviene aprovechar no solo por el incremento de la motivación del alumno, sino también porque hace más divertida la clase y promueve el compañerismo. 


\section{Agradecimientos}

Este trabajo fue realizado con el apoyo de la Iniciativa UNAM-DGAPA-INFOCABPB101719.

\section{Referencias}

Barca, A.; Porto, A.; Santorum R. \& Barca, E. (2005). Motivación académica, orientación a metas y estilos atribucionales: la escala CEAP 48. Revista de Psicología y Educación, 1(2), 103-136.

Bartle, R. A. (2005). Virtual worlds: Why people play. Recuperado el 15 de enero del 2019 de: https://bit.ly/2XkOgtt

Bartle, R. (2009). Understand the Limits of Theory. In Beyond Game Design: Nine Steps Towards Creating Better Videogames. Boston: Charles River Media.

Corzo, C. \& Reyes, C. (2017). Principales causas de reprobación de alumnos de los grupos de quinto semestre grupo seis y ocho de la escuela preparatoria número tres. (capítulo i antecedentes). Con-Ciencia Boletín Científico de la Escuela Preparatoria No. 3, 4(7). Recuperado el 01 de marzo de 2019 de: https://bit.ly/2HtHYRX

Cuevas, A., Hernández, R., Leal, B. E. y Mendoza, C. P. (2016). Enseñanza-aprendizaje de ciencia e investigación en educación básica en México. Revista Electrónica de Investigación Educativa, 18(3), 187-200.

ENCCH-UNAM (2016). Programa de estudio del Área de Ciencias experimentales Física I-II ENCCH. Recuperado el 16 de mayo de 2019 de: https://bit.ly/2Keef2V

Escaraval, J. C. \& Martín, F. (2019). Análisis bibliográfico de la gamificación en educación física. Revista Iberoamericana de Ciencias de la Actividad Física y el Deporte 8(1), 97-109.

Gallardo, J. A., \& Gallardo, P. (2018). Teorías sobre el juego y su importancia como recurso educativo para el desarrollo integral infantil. Hekademos: Revista educativa digital, (24), 41 51.
Gallego, F. J., Molina, R. \& Llorens, F. (2014). Gamificar una propuesta docente. Diseñando experiencias positivas de aprendizaje. XX Jornada sobre la enseñanza universitaria de la informática.

García, M. \& Hijón, R. (2017). Análisis para la gamificación de un curso de formación profesional. ADIE, Asociación para el Desarrollo de la Informática Educativa.

García, J., Aguilera, J. R., \& Castillo, A. (2011). Guía técnica para la construcción de escalas de actitud. Odiseo, revista electrónica de pedagogía, $8(16)$.

Godoy, M.E. (2019). La Gamificación desde una Reflexión Teórica como recurso estratégico en la Educación. Revista espacios, 40(15), 25.

Hernández, R.; Fernández, C. \& Baptista, M.P. (2014). Metodología de la investigación. México: McGraw-Hill, Interamericana editores S.A. de C.V.

López, V. \& Domènech, J. (2018). Juegos y gamificación en las clases de ciencia: ¿Una oportunidad para hacer mejor clase o para hacer mejor ciencia? Revista electrónica Ludus Scientiae, 2(1), 34-44.

Mera, J. A. (2016). Gamificación una estrategia de fortalecimiento en el aprendizaje de la ingeniería de sistemas, experiencia significativa en la Universidad Cooperativa de Colombia sede Popayán. Revista científica, 3(26), 3-11.

Montero, B. (2017). Aplicación de juegos didácticos como metodología de enseñanza: Una revisión de la literatura. Pensamiento Matemático, 7(1), 75-92.

Murillo, J.A., Carrasquero, A. \& Cañada, F. (2018). Propuesta de prácticas de laboratorio para un curso de química dirigido a adultos mayores. Revista paradigma, 39(2), 170-186.

Oliva, H.A. (2016). La gamificación como estrategia metodológica en el contexto educativo universitario. Realidad y Reflexión, 16(44), 2947.

Ortiz, A. M., Jordán, J., \& Agredal, M. (2018). Gamificación en educación: una panorámica sobre el estado de la cuestión. Educação $e$ Pesquisa, 44, 1-17. 
Rodríguez, D., Gómez, R., Bravo, M.J. \& Truyol, M. E. (2019). Aprendizaje basado en un proyecto de gamificación: vinculando la educación universitaria con la divulgación de la geomorfología de Chile. Revista Eureka sobre Enseñanza y Divulgación de las Ciencias, 16(2), 220201-220213.

Rodríguez, J. C. E., \& Martín-Acosta, F. (2019). Análisis bibliográfico de la gamificación en Educación Física. Revista Iberoamericana de Ciencias de la Actividad Física y el Deporte, 8(1), 97-109.

Sanmartí, N. \& Márquez, N. (2017). Aprendizaje de las ciencias basadas en proyectos: del contexto a la acción. Ápice. Revista de educación científica 1(1), 3-16.

Sierra, M.C. \& Fernández, M.R. (2019). Gamificando el aula universitaria. Análisis de una experiencia de Escape Room en educación superior. Revista de Estudios y Experiencias en Educación, 18(36), 105-115.

León, Ó., Martínez, L. F., \& Santos, M. L. (2019). Gamificación en educación Física: un análisis sistemático de fuentes documentales. Revista Iberoamericana de Ciencias de la Actividad Física y el Deporte, 8(1), 110-124.

Quintanal P., F. (2016). Aplicación de herramientas de gamificación en física y química de secundaria. Recuperado el: 19 de mayo de 2019 de: https://bit.ly/2Nsdt5E

Vallejo, P. M. (2011). Guía para construir cuestionarios y escalas de actitudes. Universidad Pontificia de Comillas, España.

Werbach, K. \& Hunter, D. (2012). For the Win, Philadelphia: Wharton Digital Press. 